typeset using JPSJ.sty $<$ ver.0.8 $>$

\title{
Scaling Properties of Antiferromagnetic Transition in Coupled Spin Ladder Systems Doped with Nonmagnetic Impurities 6
}

\author{
Masatoshi IMADA and Youichirou IINO \\ Institute for Solid State Physics, \\ University of Tokyo, Roppongi 7-22-1, \\ Minato-ku, Tokyo 106
}

(Received January 10, 1997 )

\begin{abstract}
We study effects of interladder coupling on critical magnetic properties of spin ladder systems doped with small concentrations of nonmagnetic impurities, using the scaling theory together with quantum Monte Carlo (QMC) calculations. Scaling properties in a wide region in the parameter space of the impurity concentration $x$ and the interladder coupling are governed by the quantum critical point ( $\mathrm{QCP}$ ) of the undoped system for the transition between antiferromagnetically ordered and spin-gapped phases. This multi-dimensional and strong-coupling region has characteristic power-law dependences on $x$ for magnetic properties such as the Néel temperature. The relevance of this criticality for understanding experimental results of ladder compounds is stressed.
\end{abstract}

KEYWORDS: spin ladder, scaling theory, quantum critical point, nonmagnetic impurity, antiferromagnetic order

Mott insulators on several geometrically designed structures of lattices are known to have the spin excitation gap from the spin singlet ground state 1 Several compounds such as $\mathrm{SrCu}_{2} \mathrm{O}_{3}$ 迸 and $(\mathrm{VO})_{2} \mathrm{P}_{2} \mathrm{O}_{7} 3^{3}$ indeed have the gap which may be attributed to the underlying ladder structure of $\mathrm{Cu}_{2} \mathrm{O}_{3}$ or $\mathrm{V}_{2} \mathrm{O}_{3}$ network. The spin$1 / 2$ Heisenberg model on a ladder given by the Hamiltonian

$$
\mathcal{H}=\sum_{\langle i, j\rangle} J_{i j} \mathbf{S}_{i} \cdot \mathbf{S}_{j}
$$

with the exchange interaction between nearest-neighbor pairs $\langle i, j\rangle$ describes low-energy spin excitations. The spin gap $A_{s}$ is as large as $0.5 \mathrm{~J}$ for the uniform strength of $J=J_{i j}$.

Recent experiments have revealed that the spin ladder compound $\mathrm{SrCu}_{2} \mathrm{O}_{3}$ shows surprisingly sensitive dependence sn small concentration of $\mathrm{Zn}$ substitution for $\mathrm{Cu}$ sites 1 . Even at the doping concentration of $\mathrm{Zn}$ as low as $x=0.01$, the system shows anomalies in the susceptibility and specific heat at the temperature $T \sim 3 \mathrm{~K}$. The $x$ dependence suggests that the compound changes from a spin-gapped insulator at $x=0$ to an insulator with an antiferromagnetic long-range order (AFLRO) below the temperature of the anomaly, $T_{N}$, which quickly increases with increasing $x$. In addition, above $T_{N}$, it shows a remarkable Curie-like behavior $\chi=C / T$ for the uniform susceptibility and a $T$-linear specific heat $C_{p}$. The sensitive dependence on $x$ and the appearance of the AFLRO have a similarity to the case of a spin-Peierls compound $\mathrm{CuGeO}_{3}$ 耐.

Since the Zn substitution plays a role to deplete the spin- $1 / 2$ local moment on a half-filled $\mathrm{Cu} d_{x^{2}-y^{2}}$ orbital, the ladder model (1) with a dilute depletion of

\footnotetext{
* to appear in J. Phys. Soc. Jpn.66(1997) No.3
}

sites has been extensively studied theoretically. Variational Monte Carlo, as well as the exact diagonalization results show that a nonmagnetic impurity induces the formation of a static spin- $1 / 2$ moment with a substantial enhancement of antiferromagnetic correlations (AFC) around the impurity 8 ). Quantum Monte Carlo (QMC) results of a single ladder have supported this result of the enhancement 9 ). This enhancement has also been suggested in other theoretical approaches for models with a spin gap 10, 11 12). The QMC results9) on ladders have further reproduced the Curie law, $\chi=C / T$ and the linear- $T$ specific heat $C_{p}=\gamma T$ with quantitative agreements of $C$ and $\gamma$ with the experimental results, when the nonmagnetic impurity concentration $x$ exceeds a rather sharp crossover concentration $x_{c} \simeq$ 0.04-0.05. However, below $x_{c}$, the local moments induced around the depleted sites are so weakly coupled that they do not explain the experimental results for $\gamma$ and the appearance of AFLRO. This is due to the fact that the effective coupling between the induced spin- $1 / 2$ moments at distance $r$ decreases exponentially as $\exp \left[-r / \xi_{0}\right]$ and this characteristic energy scale for the typical distance between neighboring induced moments becomes far below the temperature in experiments for realistic choices of the AFC length $\xi_{0}$ of a single ladder. On the contrary, the experimental results must be the consequence of strongly and antiferromagnetically coupled moments.

In this letter we study effects of interladder coupling to understand this puzzling feature. We show existence of four different regions of the critical behavior separated by two crossover lines in the parameter space of the interladder coupling $J_{L}$ and $x$. Previously employed quasi-one-dimensional (Q1D) and weak-coupling (WC) approaches from the single ladder in the literature are valid in one of the four regions, whereas we stress that a different region among the four is relevant for under- 
standing experimental indications. This region is governed by the universality of the QCP for undoped systems, where the scaling properties are characterized by strong coupling (SC) of the induced moments and the multi-dimensional (MD) criticality. We discuss the scaling properties of this antiferromagnetic transition. QMC results on coupled ladders as well as experimental indications are compared with the scaling predictions. We summarize our basic phase diagram for different scaling properties in Fig.1. Detailed definitions of WC, SC, Q1D and 3D (three-dimensional) or MD are clarified in the discussions below.

First we estimate the scaling properties of the antiferromagnetic transition and the upper bound for the Néel temperature in the Q1D region of weakly coupled (WC) ladders. This region is realized when the interladder coupling is finite but sufficiently weak. In this region, with decreasing temperature, $\xi_{0}$ within and along the ladder becomes much longer than $\xi_{L}$ for the interladder direction. The effective exchange coupling between two local moments induced at two depleted sites each depends exponentially on the distance between these depleted sites, $r$, as $J(r) \simeq J_{0} e^{-r / \xi_{0}}$ with $J_{0}$ being the nearest-neighbor exchange coupling of the uniform ladder. Here we neglect logarithmic corrections. When the impurities are doped randomly, the distribution function of the distance between two neighboring depleted sites follows the Poissontype distribution $P_{r}(r)=\frac{r}{l^{2}} e^{-r / l}$ for the concentration of depleted sites, $x=1 / 2 l$. Here, the length is scaled by the lattice constant of the ladder. From the above relations the distribution function for the amplitude of the exchange coupling between the moments at neighboring depleted sites follows

$$
P_{J}(J) \sim-\frac{\xi_{0}^{2}}{l^{2}}\left(\ln \frac{J}{J_{0}}\right) \frac{1}{J}\left(\frac{J}{J_{0}}\right)^{\frac{\xi_{0}}{l}} .
$$

When $T$ is lowered, only $J$ above the scale of $T$ can contribute to the growth of AFC. The ratio of such coupling, $R$, among all the neighboring exchange between depleted sites is estimated as

$$
R=\int_{J_{t h}}^{J_{0}} P_{J}(J) d J=1+\frac{\xi_{0}}{l}\left(\frac{J_{t h}}{J_{0}}\right)^{\frac{\xi_{0}}{l}}\left(\ln \frac{J_{t h}}{J_{0}}-\frac{l}{\xi_{0}}\right),
$$

where we take $J_{t h} \sim T$. When $x$ is low enough so that $l \gg \xi_{0}$ is satisfied, $R \simeq 1-\left(J_{t h} / J_{0}\right)^{\xi_{0} / l}$ follows. Therefore the AFC along the ladder is developed over many depleted sites only for not too small $R$ and hence only at

$$
T<T_{c r 1}=J_{0} e^{-\frac{a}{x}}
$$

with $a=\frac{1}{2 \xi_{0}} \simeq \frac{\Delta_{s}}{J_{0}}$. As we have neglected the quantum fluctuation for progressive singlet formation on the stronger bonds as is frequently discussed in the random exchange antiferromagent 10$), T_{c r 1}$ based on the above argument of the percolation threshold is certainly an upper bound temperature for the growth of a long correlation length within a ladder.

When we consider the interladder couplings $J_{L 1}$ and $J_{L 2}$, in the other two directions, the necessary condition for the growth of $3 \mathrm{D}$ correlation over many depleted sites in the WC region is similarly given from

$$
R \simeq 1-\left(\frac{T}{J_{0}}\right)^{\frac{\xi_{0}}{l}}\left(\frac{T}{J_{L 1}}\right)^{\frac{\xi_{L 1}}{l}}\left(\frac{T}{J_{L 2}}\right)^{\frac{\xi_{L 2}}{l}}
$$

with $2 l=1 / x^{1 / 3}$ as

$$
T<T_{c r 3}=J_{0} \exp \left[-\frac{\Delta_{s}}{\left(J_{0} J_{L 1} J_{L 2} x\right)^{1 / 3}}\right]
$$

at small enough $x$. The upper bound of $T_{N}$ in the $3 D$ region is given by $T_{c r 3}$.

From (14) and (6), it turns out that the Q1D treatment is always invalid at small $x$. The boundary to separate the Q1D and the 3D region is given by

$$
x_{D} \sim \frac{\sqrt{J_{L 1} J_{L 2}}}{J_{0}} .
$$

Below $x_{D}$, three dimensional correlations are developed before the correlation grows only along the ladder. Equations (4) and (6) show that, in the region of weakly coupled local moments, the upper bound of $T_{N}$ is exponentially small with an essential singular dependence on $x$. In the WC region, the upperbound of the equal-time spin structure factor $\lim _{N \rightarrow \infty} S(Q) / N \equiv \sum_{r}\left\langle s_{0}^{z} s_{r}^{z}\right\rangle e^{i Q r}$ at the staggered wave vector $Q$ should be scaled as

$$
\lim _{N \rightarrow \infty} S(Q) / N=\left\langle M_{s}\right\rangle^{2} / 3 \propto x^{2},
$$

because only the spin- $1 / 2$ local moments induced around the depleted sites contribute to the order parameter. Here $\left\langle M_{s}\right\rangle$ is the staggered magnetization.

A qualitatively different $x$ dependence of $T_{N}$ is derived in the $\mathrm{SC}$ region which is governed by the $\mathrm{QCP}$ at $J_{L 1}=J_{L 1 c}$ and $J_{L 2}=J_{L 2 c}$. This critical point is defined as the interladder coupling beyond which the AFLRO is stabilized at $T=0$ even without the nonmagnetic impurities. To discuss the scaling properties of the antiferromagnetic transition in the $\mathrm{SC}$ region, we take $\delta=J_{L c}-J_{L}, J_{L} \equiv J_{L 1}=J_{L 2}$ and $J_{L c} \equiv J_{L 1 c}=J_{L 2 c}$ for simplicity.

At $x=0$, the correlation length exponent $\nu$ and the staggered magnetization exponent $\beta$ are defined as $\xi \propto$ $\delta^{-\nu}$ and $\left\langle M_{s}\right\rangle \propto|\delta|^{\beta}$ for small $|\delta|$. The Néel temperature $T_{N}$ for $\delta<0$ is scaled from the critical point as $T_{N} \propto$ $(-\delta)^{\nu z}$ where the dynamical exponent $z$ is introduced.

When $x$ is finite but $\delta=0$, we can derive scaling exponents for $x$ dependence because $x$ is directly related with the inverse cube of the length scale. For example $T_{N}$ should be characterized by the temperature $T_{c r}^{*}$ below which the averaged correlation length $\xi$ exceeds the mean impurity distance $x^{-1 / 3}$. Below $T_{c r}^{*}$, the local moments induced around the depleted sites become strongly correlated antiferromagnetically through the overlap of the correlated region of the radius $\xi$. With lowering temperature, $\xi \sim 1 / T^{z}$ is expected. This gives the scaling

$$
T_{N} \propto x^{p}
$$

with $p=z / 3$ in three dimensions. Similarly $\left\langle M_{s}\right\rangle$ at $T=0$ is scaled as

$$
\left\langle M_{s}\right\rangle \propto x^{q}
$$

with $q=\beta / d \nu$ in $d$ spatial dimensions for $d \geq 2$. These 
scaling properties at $\delta=0$ govern the $\mathrm{SC}$ region. As compared to the WC region which shows the essentially singular dependence on $x$, characteristic scales all have power-law dependence on $x$. The crossover boundary between the $\mathrm{WC}$ and $\mathrm{SC}$ regions is given by

$$
x_{c}=\delta^{u}
$$

with $u=d \nu$. Therefore when we fix $\delta$ and increase $x$, physical quantities show a crossover from the scaling in the $\mathrm{WC}$ region to that in the $\mathrm{SC}$ region at this boundary.

The universality class of the quantum transition at $x=0$ as a function of $\delta$ in $d$ dimensions is not exactly known. However, there exists growing support for the equizalence to the $d+1$-dimensional nonlinear sigma model13. 14). The dynamical exponent $z$ is then given by $z=1$. The universality class of the four-dimensional nonlinear sigma model is characterized by the mean field exponents $\nu=0.5$ and $\beta=0.5$. Then for the exponents in the SC region, we obtain $2 p=q=2 / 3$ and $u=3 / 2$ for the $3 \mathrm{D}$ case. For comparison, we note that, in $2 \mathrm{D}$, the long-range order may appear only at $T=0$, where $q \sim 0.26$ and $u \sim 1.4$ are gbtained because $\nu \simeq 0.70$ and $\beta \simeq 0.36$ are expected 15 . It should be noted that the boundary $x_{c}$ may be rather small because $u>1$ and $x_{c}$ is extrapolated to around 0.04 even for the single 1D ladder as clarified in the QMC results9).

We next discuss quantitative aspects of the scaling properties by using the QMC results. In this letter, we show the QMC results of coupled ladders for the configuration of the two-dimensional square lattice. This structure has two basic differences from that of real compounds such as $\mathrm{SrCu}_{2} \mathrm{O}_{3}$. One is that the threedimensionality is ignored so that we cannot discuss scaling properties of $T_{N}$ directly. The other point is that the experimental lattice structure has a certain frustration on the ladder plane while it is not considered here. However, even with these drawbacks of the present model, it is still useful to understand the four different criticalities in the WC-Q1D, WC-MD, SC-Q1D and SC-MD regions with the robustness of the SC-MD region in the simplest $2 \mathrm{D}$ case. More detailed analyses with a $3 \mathrm{D}$ realistic lattice structure will be discussed elsewhere. QMC calculations have been performed by using the world line algorithm with a cluster updating method 16 and a continuous time procedure 17 . The system size is $N=L_{x} \times L_{y}$ with $L_{y}$ being the ladder direction. Several choices of linear dimensions are taken for the extrapolation between $L_{y}=32$ and 128 for $L_{y} / L_{x}=4,6$ and 8 .

We first discuss quantitative aspects at $x=0$. At $x=0$, the QCP for the antiferromagnetic transition is estimated as $J_{L c}=0.32 \pm 0.02$ as illustrated in Fig.2. For $J_{L}>J_{L c}$, the AFLRO appears at $T=0$ and $x=0$. Two methods have been employed to estimate $J_{L c}$. One is to measure the $J_{L}$ dependence of the spin gap for $J_{L}<J_{L c}$. For finite size lattices, the spin gap $\Delta_{s}$ is determined from the fitting of the uniform susceptibility $\chi_{0}$ to the form

$$
\chi_{0}(T)=A \exp \left[-\Delta_{s} / T\right] .
$$

The limit $N \rightarrow \infty$ is taken in the scaling of $\Delta_{s}$ with $1 / N^{2}$. Figure 3 shows the extrapolated values of $\Delta_{s}$.
In the other method, $J_{L}$ dependence of $\left\langle M_{s}\right\rangle$ has been examined in the region of $J_{L}>J_{L c}$. The critical point is consistent with $J_{L c}=0.32 \pm 0.02$ in both of the analyses.

When $x \neq 0$, it is expected that the AFLRO appears in all the regions of $J_{L}>0$, if $x$ is not too large. However, scaling properties for the $x$ dependence may be drastically different for different regions as we mentioned above. Here, we show $x$ dependence of $\left\langle M_{s}\right\rangle$ at $J_{L}=0.2$ and 0.3. In the following, we show results for regular distribution of impurities. The results for the random distribution show qualitatively similar behavior although the AFLRO is quantitatively suppressed. Detailed analyses will be discussed elsewhere. Note that at $x=0$, the gap extrapolated to the infinite size is estimated to be $\Delta_{s} \simeq 0.25 J_{0}$ at $J_{L}=0.2$ while $\Delta_{s} \simeq 0.05 J_{0}$ at $J_{L}=0.3$. In comparison with experimental conditions, we note that the experimentally observed gap $\Delta_{s} \sim 500 \mathrm{~K}$ in $\mathrm{SrCu}_{2} \mathrm{O}_{3} 18$, with a reasonable estimate $J_{0} \simeq 2000 \mathrm{~K}$ as deduced from the susceptibility of similar but chain-type compounds $\mathrm{Sr}_{2} \mathrm{CuO}_{3}$ and $\mathrm{SrCuO}_{2}$ 19) leads to a similar ratio of $\Delta_{s} / J_{0}$ to the case at $J_{L}=0.2$. At $J_{L}=0.3,\left\langle M_{s}\right\rangle$ appears to follow a power-law dependence $\left\langle M_{s}\right\rangle \sim a x^{q}$ with $q \sim 0.26$ for smaller $x$ as predicted in the SC-MD region of $2 \mathrm{D}$ systems. This is reasonable because $J_{L}=0.3$ is close to the critical point $J_{L c}$. The prefactor $b$ in the scaling of the crossover boundary defined by $x_{c} \sim b \delta^{1.4}$ is roughly estimated as $b \sim 0.5$ because at $J_{L}=0.2$, $x_{c} \sim 0.02$ as we see in 3 . Although the numerical results are rather preliminary, the results are not inconsistent with the scaling prediction.

We next discuss the relevance of the SC-MD region for understanding the experimental results. Because the 3D coupling is crucial to realize a nonzero $T_{N}$ and the $3 \mathrm{D}$ region dominates over the $\mathrm{Q} 1 \mathrm{D}$ and $2 \mathrm{D}$ regions for small $x$ as discussed above, the scaling properties in experimental results for small $x$ need to be analyzed by the $3 \mathrm{D}$ scaling. Although it is difficult to estimate the exponents quantitatively from the available experimental data, at least, it is consistent with a quick increase of $T_{N}$ as a function of $x$ with a wide SC region where even $x=0.01$ is involved. Based on this qualitatively promising implication, further detailed comparisons of experimental results and theoretical predictions here would be interesting. For example, clarification of pressure effects at both $x=0$ and $x \neq 0$ would be desired because rather remarkable effects are expected through the control of $\delta$ near the QCP on the basis of the above theoretical analysis. In case of $\mathrm{CaV}_{2} \mathrm{O}_{5}$, it has been reported that dilute substitution of $\mathrm{Ti}$ for $\mathrm{V}$ does not make the system antiferromagnetic 20. This difference may be understood by assuming much smaller interladder exchange coupling than in the $\mathrm{Cu}$ compound due to presumable orbital ordering of $t_{2 g}$ electrons on the $\mathrm{V}$ site.

We also note that the closeness to the QCP has an important implication for the mechanism of superconductivity under doping of itinerant holes 21]. Here, we discuss two proposals for the mechapism. One is that first proposed by one of the authors 22 ) where the carrier doping into a spin-gapped Mott insulator may directly result in a superconducting state in the region of the gap persistence. This proposal was directly applied in 
the case of ladder modelst 23). The other is the mechanism proposed for the high- $T_{c}$ cuprates, where recovery of the suppressed coherence inherent in 2D (or 3D) systems near the Mott transition results in the superconductivity 24, 25. In this mechanism, the closeness to the QCP is crucial. The nonmagnetic impurity effects have turned out to be a useful probe to measure the distance from the QCP of the magnetic order. The experimental results appear to support the relevance of the quantum critical region and hence support the latter mechanism.

In summary, effects of interladder coupling on the antiferromagnetic transition have been investigated for coupled ladder systems doped with nonmagnetic impurities. Critical properties follow different scalings depending on four different regions. They are separated by a crossover from the weak-coupling to the strong-coupling regions, as well as by a crossover from the quasi-one-dimensional to the multi-dimensional regions. The strong-coupling and three-dimensional scaling behavior governed by the quantum critical point of the undoped system dominates over a wide region, which leads to characteristic powerlaw dependences of physical quantities such as the Néel temperature on $x$. We propose that the scaling properties in this SC-3D region are relevant and important for understanding recent experimental indications in ladder compounds.

We thank H. Takagi, N. Furukawa, N. Katoh and Y. Motome for useful discussions. This work is supported by a Grant-in-Aid for Scientific Research on Priority Area "Anomalous Metallic States near the Mott Transition" from the Ministry of Education, Science and Culture. The numerical calculations were performed at the Supercomputer Center of the Institute for Solid State Physics, University of Tokyo.

[1] See for example, N. Katoh and M. Imada: J. Phys. Soc. Jpn. 64 (1995) 1437.

[2] Z. Hiroi et al.: Solid State Commun. 95 (1991) 230.

[3] D.C. Johnston et al. : Phys. Rev. B 35 (1987) 219.

[4] E. Dagotto, J. Riera and D.J. Scalapino: Phys. Rev. B 45 (1992) 5744.

[5] M. Azuma, Y. Fujishiro, M. Takano, T. Ishida, K. Okuda, M. Nohara and H. Takagi: to be published.

[6] M. Nohara, H. Takagi, M. Azuma, Y. Fujishiro and M. Takano: to be published.

[7] M. Hase, I. Terasaki and K. Uchinokura: Phys. Rev. Lett. 70 (1993) 3651.

[8] Y. Motome, N. Katoh, N. Furukawa and M. Imada: J. Phys. Soc. Jpn. 65 (1996) 1949.

[9] Y. Iino and M. Imada: J. Phys. Soc. Jpn. 65 (1996) 3728.

[10] H. Fukuyama, N. Nagaosa, M. Saito and T. Tanimoto: J. Phys. Soc. Jpn. 65 (1996) 2377.

[11] G.B. Martins, E. Dagotto and J.A. Riera: cond-mat/9605069.

[12] H.-J. Mikeska, U. Neugebauer and U. Schollwoeck: condmat/9608100

[13] S. Chakravarty, B.I. Halperin and D. Nelson: Phys. Rev. B 39 (1989) 2344.

[14] M. Troyer, M. Imada and K. Ueda: in preparation.

[15] P. Peczak et al.: Phys. Rev. B 43 (1991) 6087.

[16] H.G. Evertz, G. Lana and M. Marcu: Phys. Rev. Lett. 70 (1993) 875

[17] B.B. Beard and U.-J. Wiese: cond-mat/9602164.

[18] K. Ishida, et al. : J. Phys. Soc. Jpn. 63 (1994) 3222.

[19] N. Motoyama, H. Eisaki and S. Uchida: Phys. Rev. Lett., 76 (1996) 3212.
Fig. 1. Schematic phase diagram for the scaling properties in the plane of interladder coupling $J_{L}$ and nonmagnetic impurity concentration $x$. The quasi-one-dimensional (Q1D) and multidimensional (MD) regions are separated by the solid curve $x_{D}$ while the strong coupling (SC) and weak coupling (WC) regions are separated by the curve $x_{c}$.

Fig. 2. The spin gap $\Delta_{s}$ (triangle) and the staggered magnetization $\left\langle M_{s}\right\rangle$ (circle) as a function of $J_{L}$ scaled by $J_{0}$ at $T=0$ and $x=0$. Squares and diamonds show $\left\langle M_{s}\right\rangle$ for $T=0$ at $x=0.0156$ and 0.0208 , respectively. The dotted lines are guides for the eyes.

Fig. 3. $x$ dependence of $\left\langle M_{s}\right\rangle$ for $J_{L}=0.2$ (circle) and 0.3 (square) at $T=0$. Solid (broken) lines show the slopes for the scalings of the $\mathrm{SC}(\mathrm{WC})$ region.

[20] Y. Ueda: private communication.

[21] M. Uehara et al.: J. Phys. Soc. Jpn. 65 (1996) 2764.

[22] M. Imada: J. Phys. Soc. Jpn. 60 (1991) 1887; Phys. Rev. B 48 (1993) 550.

[23] For a review; see E. Dagotto and T.M. Rice: Science 271 (1996) 618.

[24] M. Imada: J. Phys. Soc. Jpn. 64 (1995) 2954.

[25] F.F. Assaad, M. Imada and D.J. Scalapino: Phys. Rev. Lett. 77 (1996) 4592 

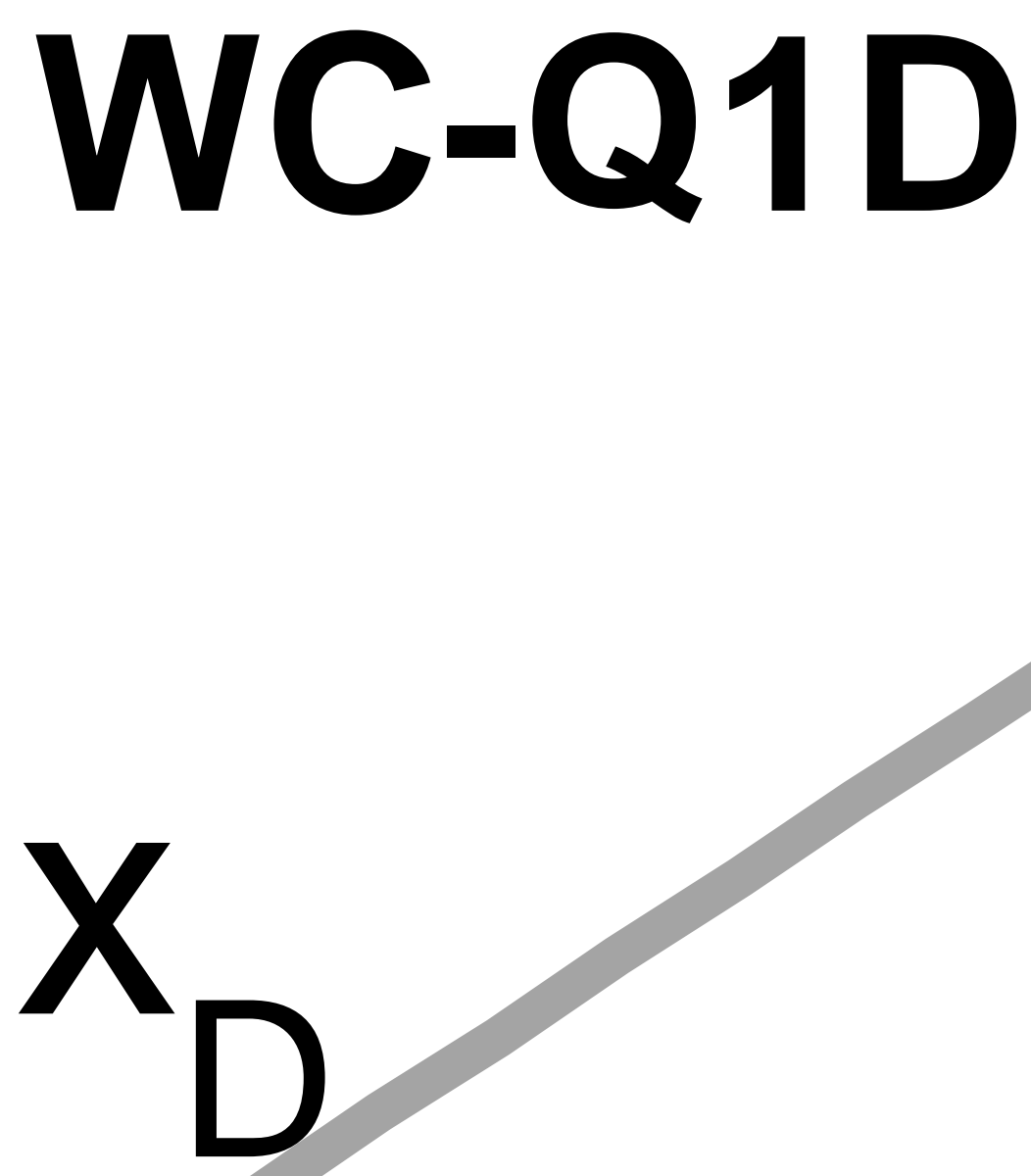

0 

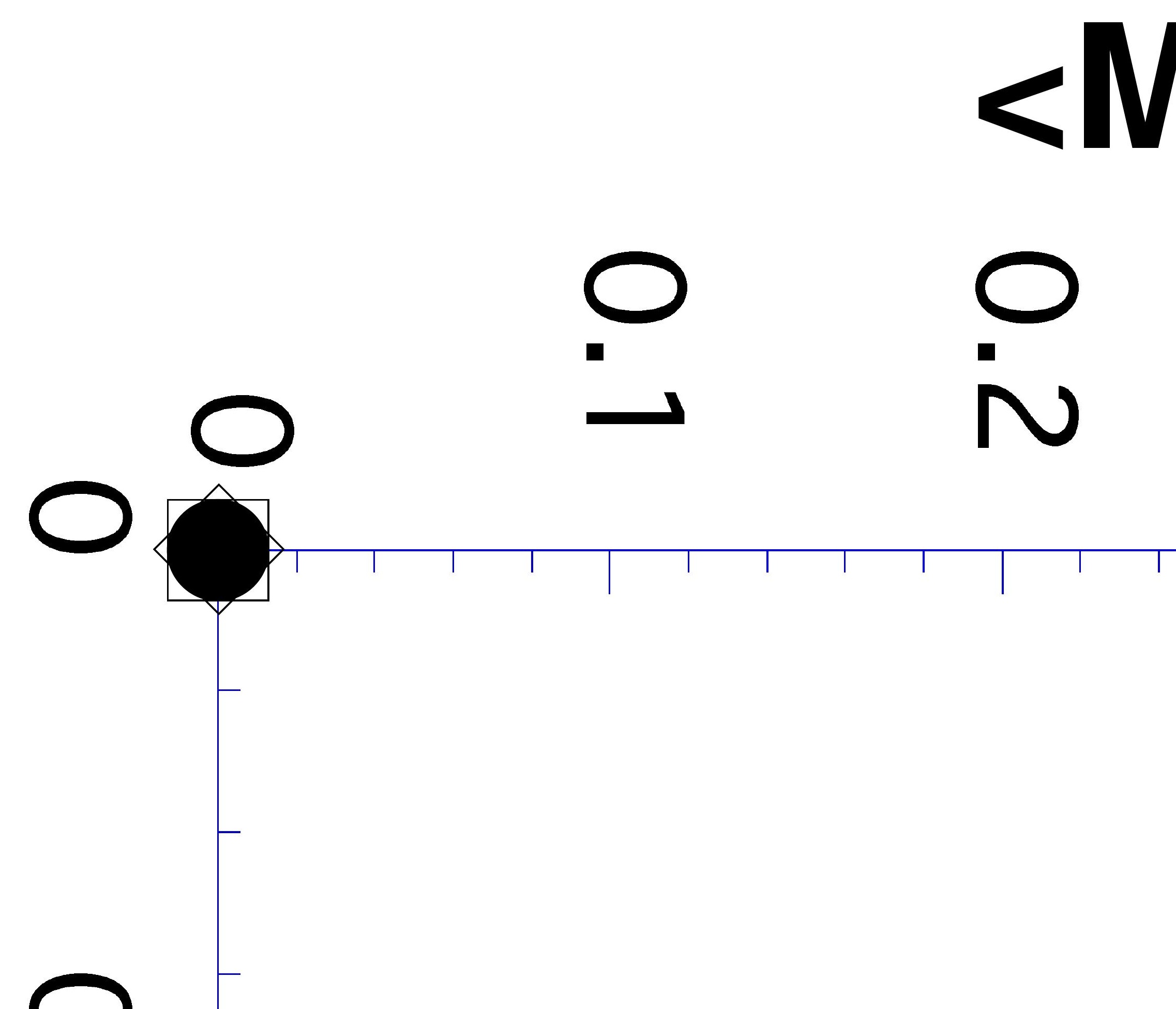


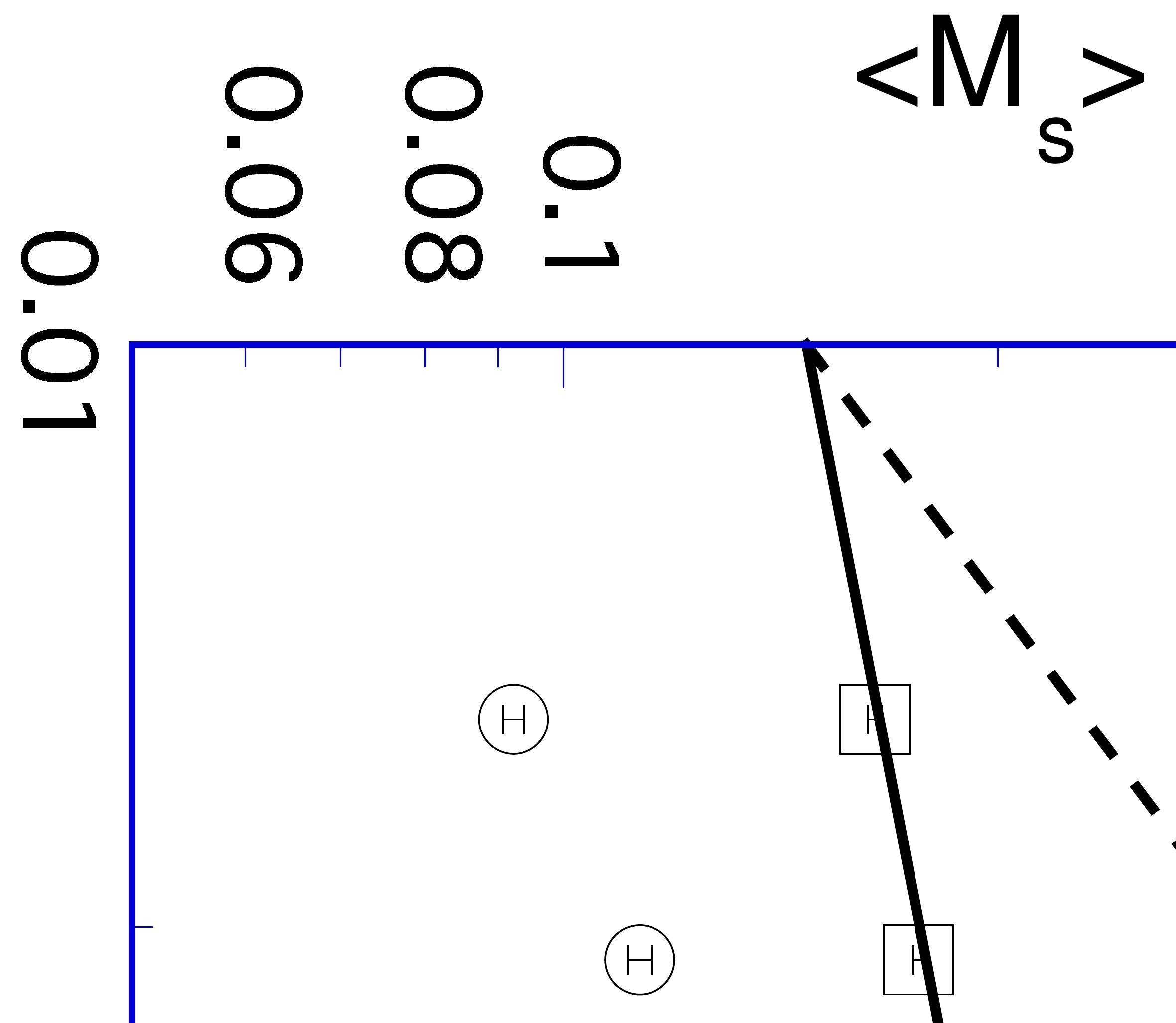

